\title{
An evolutionary game analysis of enterprise carbon emission regulation based on prospect theory
}

Hao Sun ( $\nabla 705143946 @ q q . c o m$ )

University of Shanghai for Science and Technology

\section{Guangkuo Gao}

University of Shanghai for secience and technology

\section{Zonghuo Li}

Nanjing University of Science and Technology

\section{Research Article}

Keywords: risk of deterioration of air quality, prospect theory, carbon regulation, evolutionary game, incentive paradox

Posted Date: February 2nd, 2022

DOI: https://doi.org/10.21203/rs.3.rs-1315172/v1

License: @ (i) This work is licensed under a Creative Commons Attribution 4.0 International License. Read Full License 


\title{
An evolutionary game analysis of enterprise carbon emission regulation based on prospect theory
}

\author{
Hao Sun ${ }^{1, *}$, Guangkuo $\mathrm{Gao}^{2}$, Zonghuo $\mathrm{Li}^{3}$
}

\begin{abstract}
Aiming at the problem that the government regulates the carbon emission of enterprises, this paper introduces the prospect theory to correct the revenue function of government and enterprise, considering the risk of air quality deterioration, built an evolutionary game model of government and enterprise based on prospect theory. According to the weight of the two sides on the probability of air quality deterioration under the equilibrium state, the risk of air quality deterioration is divided into three levels: low, medium and high. Analyzed the evolution strategy of government and enterprise under different levels. The results show that when the risk of air quality deterioration is low, the government tends to negative regulation and enterprises adopt traditional production. When the risk of air quality deterioration is high, government tend to be positive in regulation and enterprises are inclined to low-carbon production at the beginning. However, with the implementation of enterprises low-carbon production, government departments will turn to negative regulation. At the same time, there will be incentive paradox phenomenon in the process of government regulation of carbon emissions.In the long run, it is more effective to strengthen the punishment of government departments' ineffective supervision than to punish enterprises.
\end{abstract}

Key words: risk of deterioration of air quality; prospect theory; carbon regulation; evolutionary game; incentive paradox

\section{Introduction}

In the context of global warming, green and low-carbon cycle development has become the inevitable trend of the development of all countries in the world. In the past decade, China's total carbon emissions accounted for more than $30 \%$ of the global total, $20 \%$ of the population's death is directly or indirectly related to air quality,

1 Hao Sun*, Business School, University of Shanghai for Science and Technology, Shanghai, China, E-mal: 705143946@qq.com, telephone :+8618769900126;

2 Guangwan Gao, Business School, University of Shanghai for Science and Technology, Shanghai, China;

3 Zonghua Li, School of Economics and Management, Nanjing University of Science and Technology, Nanjing, China. 
and air pollution causes great harm to people's health ( $\mathrm{Li}$ and Sun,2018). For this purpose, Xi J.P, the general secretary of China, has put forward the concept of ecological and green development, "We prefer clear water and green mountains to mountains of gold and silver". In order to control carbon emissions, the central government has implemented a series of measures, such as establishing an emissions trading market, imposing a carbon tax, and subsidizing the new energy industry.

The continuous carbon emission of enterprises may lead to the deterioration of air quality which affect people's life quality seriously and reduce social benefits. Meanwhile, the enterprises will also be punished by the government and suffer loss.According to Porter's hypothesis,low-carbon production can help enterprises avoid the risk of environmental penalty and offset the input cost of enterprises. Finally, achieve a win-win situation between environmental protection and profit targets(Porter and Linde, 1995; L and T, 2012). However, the limited funds used to the introduction of environmental protection equipment will increase production costs (Clarkson et al., 2004) and reduce production efficiency (Gray and Shadbegian, 1993) of enterprises in short term. So enterprises prefer to determine current profits and their own economic benefits that they are difficult initiative to take the their limited financial resources for environmental investment with low short-term economic benefits and great uncertainty in the future (Orsato, 2006; S and Wang, 2013). Enterprises will weigh low carbon production input against economic benefits to choose the most favorable production mode and give up environmental protection speculation for profit(Qin,2017). On the other hand, local governments are the main body of carbon emission supervision and should actively supervise the carbon emission of enterprises in the region. However,government supervision requires more manpower, material resources and financial costs. Meanwhile, the fiscal tax contributed by enterprises' business activities is conducive to local economic growth, which in turn contributes to the realization of local government's governing target performance. From this perspective, local governments also lack sufficient motivation to urge polluting enterprises to carry out low-carbon production, resulting in negative regulation (Zhang,2014; Pan and Yang ,2019). Thus, it can be seen that carbon emission regulation is a game process between government and enterprises.It is important to accurate understanding of the behavior choice and mutual influence mechanism between government and enterprises in the game process to reduce enterprises' carbon emission and improve environmental quality. 
Under the condition of information asymmetry, both government departments and enterprises are limited rational subjects, and they may have cognitive and value judgment biases in the process of strategic selection. Though evolutionary game analysis can effectively relax the rational assumption of game players, but there are subjective cognition bias of profit and loss in the evolutionary game between government and enterprise. This kind of cognition will affect the evolution trend of the game between government and enterprise. Prospect theory is developed by revising the maximum subjective expected utility theory, its proposal enriches the research in behavioral science and decision analysis( $\mathrm{Li}$ and $\mathrm{Li}, 2017$; Gu, Zhang and Li, 2020). So Using evolutionary game theory and prospect theory to analyze government and enterprise behaviors in carbon emission regulation can make the research more consistent with the real behavior.

This paper has three main contributions: (1)Though introduce prospect theory into the game process of carbon emission regulation, we considering the psychological expectations and value perception of the government and enterprises on profit and loss, it is more in line with the actual behavior. (2) According to the equilibrium point of air quality deterioration divided air quality deterioration risk into three levels, the strategy evolution of government and enterprises at each level was analyzed respectively. (3)We considering the incentive paradox that may occur in the regulatory process, analysis the incentive model for government and enterprises from the perspective of long - term and short - term.

The rest of this article is organized as follows.Section 2 reviews the relevant literature.Section 3 introduces the basic theories and models of carbon emission regulation, including prospect theory, evolutionary game model, conditional hypothesis and benefit function.In section 4, the equilibrium strategy and incentive paradox of the game model are analyzed, including the basic evolution strategy analysis of government departments and enterprises under different air quality and the incentive paradox analysis. In section 5, numerical simulation is carried out and the simulation results are described. Finally, the paper summarizes the whole paper and puts forward the future research direction.

\section{Literature review}

Many scholars have conducted researches on carbon emission. In the study of corporate emissions(wang et al., 2020; Jonathan et al., 2018), the scholars proposed that manufacturers and retailers cooperate vertically to reduce emissions and build a 
mission-oriented systematic low-carbon industrial strategy. Banet et al.(2017) discussed the necessity of government to use tax as federal Europe ETS supplementary tools under the background of low price subsidies. Ni Juan(2016)draw lessons from international experience, put forward the joint application of carbon tax and carbon emission trading mechanism suitable for China's national conditions. On carbon emission legislation research, Li Yanfang et.al.(2015)studied the legal position and legislative path of carbon. Zhu Xiaohui et al.(2020)put forward the improvement measures of China's legal system at the existing problems in carbon emission trading practice. To sum up, scholars have conducted researches on carbon emissions problem from taxation, emission reduction and legislation, which have provided theoretical basis for the regulation of government departments.

In the study of carbon emission regulation, due to involving the characteristics of the bounded rational behavior of main body, government and enterprises can not find the optimal strategy in the process of strategic choice at the beginning, but after constantly adjust. Evolutionary game can effectively offset the limitation of the traditional game theory, Wang Wenju et al.(2019)constructed an evolutionary game model of emission reduction behaviors of local governments and enterprises under the carbon tax mechanism, and analyzed the influence of carbon tax rate and various factors of both sides on the strategic choice. Based on the perspective of third-party supervision, Zhang Kaize et al.(2020)studied the evolution strategies of the government and enterprises in carbon emission regulation.

Although evolutionary game analysis can effectively relax the rational assumption of game players, however classical evolutionary game methods still lack the construction of participants' subjective cognition, especially the subjective perception of gains and losses in different situations faced by enterprises. Prospect theory is the research achievement of psychology and behavioral science. It is developed by revising the maximum subjective expected utility theory, and its proposal enriches the research in behavioral science and decision analysis ( $\mathrm{Li}$ and Li,2017; gu, Zhang and Li, 2020). Scholars have applied prospect theory to many fields such as management science and economics, Wang Zhiying et al.(2020)studied the evolution of panic buying events considering the perceived value of the public.Chirag Surti et al.(2020)studied the performance of prospect theory and feedback based on the newsvendor model model.Gong $\mathrm{Pu}$ et al.(2020)applied prospect theory to the search model and explored the problem of seller's pricing in the 
housing market. In the research on the combination of prospect theory and evolutionary game, Li Zonghua et al.(2017)used prospect theory to establish the supervision perception benefit matrix of enterprises and local governments on low-carbon production, and analyzed the strategy evolution game process of enterprises and local governments. However, it gives the perceived benefits to both sides of the game by direct hypothesis, and does not analyze the specific formation process.During the game, the strategies of the two players will change with the change of the environment. Wang Junjin et al.(2018) consider the environmental impact,studied the evolution of strategies of supervisors and transporters in shipping supervision under different risk levels. There are usually paradoxes in management. Some scholars have studied the paradoxes in the incentive process. Zhao Laijun et al.(2005) studied environmental law enforcement based on the paradox of incentives.Sinn et al.(2008) put forward the green paradox theory, believing that the implementation of carbon emission reduction policies may also lead to the acceleration of fossil energy exploitation, thus worsening environmental conditions. Liu Weibing et al(2019) analyzed the incentive paradox of corruption based on the perspective of game theory.

To sum up, the government's regulation of carbon emission enterprises is a game process between the two sides. Due to the limitations in rational cognition, analysis and action ability, government departments and enterprises are both bounded rational, and their strategic choice mainly depends on the psychological expectation and value perception of profit and loss (Zhao,Z.B and M, Q.P,2018). Therefore, based on the conventional evolutionary game model, this paper USES the prospect theory to correct the gains and losses of both parties in the game payment matrix.Considering the influence of the deterioration degree of air quality on the strategy choice of government and enterprise, and the strategy evolution mechanism of the two sides was discussed under different circumstances. At the same time, combining incentive paradox theory analysis regulation of emissions from the perspective of long-term and short-term, finally using the numerical simulation describes the enterprise game under the different states of evolution strategy(Tang et al., 2020; Du et al., 2016; Yang et al.,2020).

\section{3 dynamic game of carbon emission regulation}

\subsection{Problem description and model assumptions}

Government departments and enterprises are limited rational subjects, they have 
their own strategic choices in terms of the perception of gains and losses. Under the condition that air quality does not deteriorate, the benefits (social benefits) of passive regulation by government departments are $\pi_{1}$, the additional costs of active regulation are $C_{1}$. The enterprise's income from traditional production is $\pi_{2}$, and the extra cost required for low-carbon production is $C_{2}$. When the traditional production of the enterprise is investigated and punished by the government department, amount of the fine is $D$; When the air quality deteriorates, the income of the government is $-m$, the enterprise is $-t$.

Hypothesis1: There are only two parties in the game, government regulators and carbon emission enterprises, both of them are bounded rational. They choose strategies under uncertain circumstances. The optimal profit strategy cannot be found through one game, but must be realized through constant adjustment.

Hypothesis2: Government and enterprises have two choice strategies:active regulation and passive regulation,low-carbon production and traditional production. As long as either government departments or enterprises choose to take a positive attitude, there will be no risk of deterioration of air quality.

Hypothesis3: Enterprises and government departments are more sensitive to local air quality and the strategies of the other side.In order to maximize their own interests, opportunistic behaviors may exist in two parties.

Hypothesis4: The strategic choice of the two sides is based on the perception of their respective returns in the game.Both sides have the same risk avoidance coefficient and sensitivity. When the air pollution does not deteriorate, both sides can obtain normal returns.

Hypothesis5: Only the government negative regulation and the enterprise traditional production are carried out at the same time, the air quality deterioration is likely to happen, assuming deterioration probability is $p$.

\subsection{Construction of perceived return matrix}

The decision-maker's attitude towards risk is determined by the value function and the weight function. The prospect value $V$ represents the judgment made by decision makers based on their psychological feelings, as shown below:

$$
V=\sum_{i=1}^{n} w\left(p_{i}\right) v\left(\Delta x_{i}\right)
$$

In equation (1): $w\left(p_{i}\right)$ stands for decision weight; $v\left(\Delta x_{i}\right)$ represents the value perceived by the decision maker subjectively. The subjective perceived value function proposed by Tversky et al is a power function, The expression is: 


$$
v(\Delta x)=\left\{\begin{array}{cr}
\Delta x^{a 1}, & x \geq 0 \\
-\lambda(-\Delta x)^{a 2}, & x<0
\end{array}\right.
$$

The decision weight is the subjective judgment of the decision maker, according to the probability of the occurrence of the event. The expression is:

$$
w\left(p_{i}\right)=\frac{p_{i}^{r}}{\left(p_{i}^{r}+\left(1-p_{i}\right)^{r}\right)^{r}}
$$

Among them, $\alpha_{1}$ and $\alpha_{2}$ represent the sensitivity coefficient of decision makers to risks. The larger their values are, the more decision makers are inclined to take risks. $\lambda$ is the risk aversion coefficient, the larger value it is, the more risk aversion it is.Using the experimental method of Tversky, $\alpha=a_{1}=a_{2}$ is approximately0.88, risk avoidance coefficient $\lambda$ is approximately2, the regulation coefficient $r$ is approximately 0.61 .

According to (3), The weight function of air quality deterioration occurs is $w(p)$, the weight function of air quality deterioration does not occur is $w(1-p)$. So the perceived benefits of matrix of the government and enterprises shown in table 1:

Table 1 Perceived revenue matrix of government departments and carbon emitting enterprises

\begin{tabular}{llcc}
\hline game-agent & government sector & $\begin{array}{l}\text { Positive } \\
\text { regulation }(x)\end{array}$ & $\begin{array}{l}\text { Negative } \\
\text { regulation }(1-x)\end{array}$ \\
\hline \multirow{2}{*}{$\begin{array}{l}\text { manufacturing } \\
\text { enterprise }\end{array}$} & Low carbon & $V_{1}-C_{1}$ & $V_{1}$ \\
& Troduction $(y)$ & $V_{2}-C_{2}$ & $V_{2}-C_{2}$ \\
\cline { 2 - 4 } & production $(1-y)$ & $V_{1}-C_{1}$ & $V_{4}$ \\
\hline
\end{tabular}

1)In the case of positive supervision by government departments and low carbon production by enterprises. The total revenue of government departments is $V_{1}-C_{1}$, $V_{1}$ represents the social benefits perceived by government departments. The total revenue of the enterprise is $V_{2}-C_{2}, V_{2}$ represents the perceived revenue obtained by the enterprise; In this case, the possibility of air quality deterioration is 0 . Therefore, we are taking 0 as the reference point, so the perceived benefits of the government and enterprises can be obtained from Equation (1) :

$$
\begin{aligned}
& V_{1}=w(1-p) \times v\left(\pi_{1}-0\right)+w(p) \times v(0-m)=v\left(\pi_{1}\right)>C_{1} \\
& V_{2}=w(1-p) \times v\left(\pi_{2}-0\right)+w(p) \times v(0-t)=v\left(\pi_{2}\right)>C_{2}
\end{aligned}
$$

2)In the case of low carbon production by production enterprises and negative regulation by government departments, the air pollution will also not occur. Due to the government supervision department does not invest the supervision cost, so the total revenue of government is $V_{1}$, while the total revenue of enterprise is $V_{2}-C_{2}$.

3)when the enterprise take traditional production under positive supervision by 
the government department, the enterprise's behavior will be quickly discovered and stopped by the government regulatory department. So there is no risk of air quality deterioration under such circumstances. After being investigated and punished by the government department, the enterprise not only can not obtain the expected income $\pi$, but also needs to pay a penalty $D$, this moment the total income of the enterprise is $V_{3}$, while the revenue of the government department is social benefit, the fines collected are not treated as revenue, so the total income of the government department is still $V_{1}-C_{1}$. Taking 0 as the reference point, the perceived income $V 3$ of the enterprise can be obtained from Equation (1) :

$$
V_{3}=w(1-p) \times v(-D-0)+w(p) \times v(0-t)=v(-D)>C_{2}
$$

4)When the enterprises take traditional production under negative regulation of government departments, the possibility of air quality deterioration is $p$. In the case of air quality deterioration, social benefits will be greatly reduced, the superior government will conduct interviews and hold local governments accountable. At this point, the revenue obtained by the government department is $V_{4}$. When the government suffers losses, it will turn to strictly regulate the enterprise, the enterprise will stop production for rectification. At this time, the total income of the enterprise is $V_{5}$. Taking 0 as the reference point, perceived benefits of the government and enterprises as follows:

$$
\begin{aligned}
& V_{4}=w(1-p) \cdot v\left(\pi_{1}-0\right)+w(p) \cdot v(0-m)=w(1-p) \cdot v\left(\pi_{1}\right)+w(p) \cdot v(-m) \\
& V_{5}=w(1-p) \cdot v\left(\pi_{2}-0\right)+w(p) \cdot v(0-t)=w(1-p) \cdot v\left(\pi_{2}\right)+w(p) \cdot v(-t)
\end{aligned}
$$

\section{Analysis of evolutionary game model based on prospect theory}

Because the difference between $w(1-p)$ and $1-w(p)$ is small, for the convenience of subsequent calculation, we assume $w(1-p)=1-w(p)$. According to the revenue of government and enterprises in Table 1, the replication dynamic equation of both parties as follows:

$$
\begin{aligned}
& F(x)=x(1-x)\left\{y\left[-w(p)\left(v\left(\pi_{1}\right)-v(-m)\right)\right]-C_{1}+w(p)\left[v\left(\pi_{1}\right)-v(-m)\right]\right\} \\
& F(y)=y(1-y)\left\{x\left[-v(-D)+v\left(\pi_{2}\right)+w(p)\left(v(-t)-v\left(\pi_{2}\right)\right)\right]-C_{2}+w(p)\left[v\left(\pi_{2}\right)-v(-t)\right]\right\}
\end{aligned}
$$

3.1 Strategy analysis of government departments

When $F(x)$ satisfies $F(x)=0$ and $F^{\prime}(x)<0$, there is an evolutionary stable strategy. Take the derivative of $F(x)$ with respect to $x$ is:

$$
F^{\prime}(x)=(1-2 x)\left\{y\left[-w(p)\left(v\left(\pi_{1}\right)-v(-m)\right)\right]-C_{1}+w(p)\left[v\left(\pi_{1}\right)-v(-m)\right]\right\}
$$

When $F(x)=0$, the equilibrium solution can be obtained as:

$$
x_{1}=0, x_{2}=1, y^{*}=\frac{-C_{1}+w(p)\left[v\left(\pi_{1}\right)-v(-m)\right]}{w(p)\left[v\left(\pi_{1}\right)-v(-m)\right]}
$$


The evolution strategy can be discussed in the following:

1) In case $y^{*}<0$, namely $\frac{-C_{1}+w(p)\left[v\left(\pi_{1}\right)-v(-m)\right]}{w(p)\left[v\left(\pi_{1}\right)-v(-m)\right]}<0$

Due to $w(p)\left[v\left(\pi_{1}\right)-v(-m)\right]>0$, we know $-C_{1}+w(p)\left[v\left(\pi_{1}\right)-v(-m)\right]<0$, we can get $w(p) \in\left(0, \frac{C_{1}}{v\left(\pi_{1}\right)-v(-m)}\right)$. At this point $y>y^{*}$, from $F(0)=0, F^{\prime}(0)<0$, we have that $x_{1}=0$ is an evolutionary stability strategy, it indicate that in this case, if enterprises carry out low carbon production at a level higher than $\frac{-C_{1}+w(p)\left[v\left(\pi_{1}\right)-v(-m)\right]}{w(p)\left[v\left(\pi_{1}\right)-v(-m)\right]}$, the government will evolve into negative regulation.

2) In Case $0 \leq y^{*}<1$, namely $0<\frac{-C_{1}+w(p)\left[v\left(\pi_{1}\right)-v(-m)\right]}{w(p)\left[v\left(\pi_{1}\right)-v(-m)\right]}<1$

From $w(p)\left[v\left(\pi_{1}\right)-v(-m)\right]>0$, we have $-C_{1}+w(p)\left[v\left(\pi_{1}\right)-v(-m)\right]>0$, so we get $w(p) \in\left(\frac{C_{1}}{v\left(\pi_{1}\right)-v(-m)}, 1\right)$. When $y<y^{*}$, from $F(1)=0$ and $F^{\prime}(1)<0$, we get $x_{1}=1$ is a stable evolution strategy, it indicate that if carbon emitting enterprises carried out low carbon production at a level lower than $\frac{-C_{1}+w(p)\left[v\left(\pi_{1}\right)-v(-m)\right]}{w(p)\left[v\left(\pi_{1}\right)-v(-m)\right]}$, government will evolve to active regulation. When $y>y^{*}$, from $F(0)=0, F^{\prime}(0)<0$, we have $x_{1}=0$ is an evolutionary stability strategy. In this case, if enterprises carry out low-carbon production at a level higher than $\frac{-C_{1}+w(p)\left[v\left(\pi_{1}\right)-v(-m)\right]}{w(p)\left[v\left(\pi_{1}\right)-v(-m)\right]}$, the government will evolve into negative regulation. When $y=y^{*}$, from $F(x)=0, F^{\prime}(x)=0$, we have that all $x$ is a stable equilibrium state, it means that the government will get the same benefits no matter which strategy it adopts.

3)In Case $y^{*}>1$, namely $\frac{-C_{1}+w(p)\left[v\left(\pi_{1}\right)-v(-m)\right]}{w(p)\left[v\left(\pi_{1}\right)-v(-m)\right]}>1$

From $w(p)\left[v\left(\pi_{1}\right)-v(-m)\right]>0,-C_{1}+w(p)\left[v\left(\pi_{1}\right)-v(-m)\right]>w(p)\left[v\left(\pi_{1}\right)-v(-m)\right]>0$, we get $C_{1}<0$, so this situation does not exist.

To sum up, when $w(p) \in\left(0, \frac{C_{1}}{v\left(\pi_{1}\right)-v(-m)}\right), x=0$ is the evolutionary stability strategy; When $w(p) \in\left(\frac{C_{1}}{v\left(\pi_{1}\right)-v(-m)}, 1\right)$,if $y>y^{*}, x=0$ is the stable evolution strategies; If $y<y^{*}, x=1$ is the stable evolution strategies

\subsection{Strategy analysis of carbon emission enterprises}

When $F(y)$ satisfies $F(y)=0$ and $F^{\prime}(y)<0$, it is an evolutionary stabilization 
strategy, take the derivative of $F(y)$ with respect to $y$ is:

$$
F^{\prime}(y)=(1-2 y)\left\{x\left[-v(-D)+v\left(\pi_{2}\right)+w(p)\left(v(-t)-v\left(\pi_{2}\right)\right)\right]-C_{2}+w(p)\left[v\left(\pi_{2}\right)-v(-t)\right]\right\}
$$

When $F(y)=0$, we can be obtained:

$$
y_{1}=0, \quad y_{2}=1, \quad x^{*}=\frac{C_{2}-w(p)\left[v\left(\pi_{2}\right)-v(-t)\right]}{-v(-D)+v\left(\pi_{2}\right)+w(p)\left[v(-t)-v\left(\pi_{2}\right)\right]}
$$

The evolution strategy can be discussed in the following :

1) In the case $x^{*}<0$, namely $\frac{C_{2}-w(p)\left[v\left(\pi_{2}\right)-v(-t)\right]}{-v(-D)+v\left(\pi_{2}\right)-w(p)\left[v\left(\pi_{2}\right)-v(-t)\right]}<0$

(1)When $-v(-D)+v\left(\pi_{2}\right)-w(p)\left[v\left(\pi_{2}\right)-v(-t)\right]>0, C_{2}-w(p)\left[v\left(\pi_{2}\right)-v(-t)\right] \leq 0$, we get $C_{2}-w(p)\left[v\left(\pi_{2}\right)-v(-t)\right] \leq 0$, it conform to the hypothesis $v\left(\pi_{2}\right)>C_{2}$;

(2)When $-v(-D)+v\left(\pi_{2}\right)-w(p)\left[v\left(\pi_{2}\right)-v(-t)\right]<0, C_{2}-w(p)\left[v\left(\pi_{2}\right)-v(-t)\right]>0$, we get $v\left(\pi_{2}\right)<C_{2}$, It not conform to the hypothesis, so there is no such possibility.

Therefore, from $x>x^{*}$, we have $F(1)=0, F^{\prime}(1)<0$, so we can be concluded that $y=1$ is the stable point of evolution, it indicate that in this case, if the level of government positive regulation is greater than $\frac{C_{2}-w(p)\left[v\left(\pi_{2}\right)-v(-t)\right]}{-v(-D)+v\left(\pi_{2}\right)+w(p)\left[v(-t)-v\left(\pi_{2}\right)\right]}$, the enterprises will evolve to low-carbon production.

2) In case $0<x^{*}<1$, namely $0<\frac{C_{2}-w(p)\left[v\left(\pi_{2}\right)-v(-t)\right]}{-v(-D)+v\left(\pi_{2}\right)-w(p)\left[v\left(\pi_{2}\right)-v(-t)\right]}<1$

(1)When $-v(-D)+v\left(\pi_{2}\right)-w(p)\left[v\left(\pi_{2}\right)-v(-t)\right]>0$, $-v(-D)+v\left(\pi_{2}\right)-v(p)\left[v\left(\pi_{2}\right)-v(-t)\right]>C_{2}-v(p)\left[v\left(\pi_{2}\right)-v(-t)\right]>0$, we have $C_{2}<v\left(\pi_{2}\right)-v(-D)$, that satisfies hypothesis $v\left(\pi_{2}\right)>C_{2}$, so we get $w(p) \in\left(0, \frac{C_{2}}{v\left(\pi_{2}\right)-v(-t)}\right)$.

(2) When $-v(-D)+v\left(\pi_{2}\right)+w(p)\left[v(-t)-v\left(\pi_{2}\right)\right]<0, C_{2}-w(p)\left[v\left(\pi_{2}\right)-v(-t)\right]<0$, we know that $C_{2}>v\left(\pi_{2}\right)-v(-t)$ does not meet the condition $v\left(\pi_{2}\right)>C_{2}$ in the hypothesis, so the situation does not exist.

$$
\text { Under } w(p) \in\left(0, \frac{C_{2}}{v\left(\pi_{2}\right)-v(-t)}\right) \text {, when } x>x^{*} \text {, from } F(1)=0, F^{\prime}(1)<0 \text {, we }
$$

have $y=1$ is the evolutionary stable point, it indicate that if the level of government 
regulation is higher than $\frac{C_{2}-w(p)\left[v\left(\pi_{2}\right)-v(-t)\right]}{-v(-D)+v\left(\pi_{2}\right)+w(p)\left[v(-t)-v\left(\pi_{2}\right)\right]}$, enterprises will evolve to low-carbon production. When $x<x^{*}$, when $F(0)=0, F^{\prime}(0)<0$, we have $y_{1}=0$ is the evolutionary stable point, it indicate that if the supervision by government less than $\frac{C_{2}-w(p)\left[v\left(\pi_{2}\right)-v(-t)\right]}{-v(-D)+v\left(\pi_{2}\right)+w(p)\left[v(-t)-v\left(\pi_{2}\right)\right]}$, enterprises will evolve to the traditional production. When $x=x^{*}$, from $F(y)=0, F^{\prime}(y)=0$, all $y$ are in a stable equilibrium state, it indicate that if the government regulation level is equal to $\frac{C_{2}-w(p)\left[v\left(\pi_{2}\right)-v(-t)\right]}{-v(-D)+v\left(\pi_{2}\right)+w(p)\left[v(-t)-v\left(\pi_{2}\right)\right]}$, enterprise choose low-carbon production or traditional production with the same income.

3)In case $x^{*}>1$, namely $\frac{C_{2}-w(p)\left[v\left(\pi_{2}\right)-v(-t)\right]}{-v(-D)+v\left(\pi_{2}\right)+w(p)\left[v(-t)-v\left(\pi_{2}\right)\right]}>1$

(1)When $-v(-D)+v\left(\pi_{2}\right)-w(p)\left(-v\left(\pi_{2}\right)-v(-t)\right)>0, C_{2}-w(p)\left(v\left(\pi_{2}\right)-v(-t)\right)>0$, We have $C_{2}-w(p)\left(v\left(\pi_{2}\right)-v(-t)\right)>0$, which not satisfies the condition $v\left(\pi_{2}\right)>C_{2}$ in hypothesis, so this situation not exist.

(2) When $-v(-D)+v\left(\pi_{2}\right)-w(p)\left(-v\left(\pi_{2}\right)-v(-t)\right)<0$, we have $C_{2}-w(p)\left(v\left(\pi_{2}\right)-v(-t)\right)<0$, $C_{2} \leq v\left(\pi_{2}\right)-v(-D)$, which satisfies the hypothesis condition $v\left(\pi_{2}\right)>C_{2}$, Therefore, we have $w(p) \in\left(\frac{C_{2}}{v\left(\pi_{2}\right)-v(-t)}, 1\right)$.

Under $w(p) \in\left(\frac{C_{2}}{v\left(\pi_{2}\right)-v(-t)}, 1\right)$, When $x=1<x^{*}$, There is always $F(1)=0$, $F^{\prime}(1)<0$, so we have $y=1$ are evolutionary stable points, it indicate that when the level of government regulation reaches1, enterprises will evolve to low-carbon production.

To sum up, when $w(p) \in\left(0, \frac{C_{2}}{v\left(\pi_{2}\right)-v(-t)}\right)$, if $x>x^{*}$, then $y=1$ is a stable evolution strategy; If $x<x^{*}$, then $y=0$ is a stable evolution strategy; If $x=x^{*}$, then any level within the value range of $y$ is stable; When $w(p) \in\left[\frac{C_{2}}{v\left(\pi_{2}\right)-v(-t)}, 1\right)$, $y=1$ is a stable evolution strategy.

\subsection{Analysis of evolution strategies under different conditions}

According to the strategy selection probability of enterprises and government, 
assuming the possibility of air quality deterioration is $p$, Then the probability weight of government and enterprise perception is $w(p)$. Using $w_{1}(p)=\frac{C_{1}}{v(\pi)-v(-m)}$ and $w_{2}(p)=\frac{C_{2}}{v\left(\pi_{2}\right)-v(-t)}$ to divide the probability of air quality deterioration into low, medium and high three levels on $(0,1)$. Then the evolution strategies of government and enterprise at each level are analyzed respectively in the following two situations:

The first case: $0<\frac{C_{1}}{v\left(\pi_{1}\right)-v(-m)}<\frac{C_{2}}{v\left(\pi_{2}\right)-v(-t)}<1$

1)when $w(p) \in\left(0, \frac{C_{1}}{v\left(\pi_{1}\right)-v(-m)}\right)$, The production strategy selection of enterprises depends on the probability that the government regulators choose supervision.namely $x>x^{*}, y=1$ and $x<x^{*}, y=0$, from the above analysis, we can see in such circumstances, the government will choose negative regulation, namely $x=0$, Due to the $x^{*}>0$, we have know $x<x^{*}$, so the enterprise will choose traditional production strategy, Therefore, the evolutionary strategy of both sides is $x=0, y=0$.

2)when $w(p) \in\left(\frac{C_{1}}{v\left(\pi_{1}\right)-v(-m)}, \frac{C_{2}}{v\left(\pi_{2}\right)-v(-t)}\right)$, The production strategy selection of enterprises also depends on the probability that the government regulators choose supervision, namely $x>x^{*}, y=1$ and $x<x^{*}, y=0$. Regulation strategy selection of government changes with production strategy of enterprise,namely, $y>y^{*}$, $x=1$ and $y<y^{*}, x=0$, so the strategic choice of government and enterprise infinite cycle.

3) When $w(p) \in\left(\frac{C_{2}}{v\left(\pi_{2}\right)-v(-t)}, 1\right)$, it can be seen from the above analysis, enterprise choose low-carbon production strategies. The choice of regulatory strategy of government depends on the production strategy of enterprises, namely $y>y^{*}$, $x=0$ and $y<y^{*}, x=1$.From $y=1$, we can get $y>y^{*}$. so we have $x=0$ is evolutionary stability point, namely government department will choose the negative regulation strategy. Therefore, the evolution strategy of government and enterprise is $x=0, y=1$. 
The second case: $0<\frac{C_{2}}{v\left(\pi_{2}\right)-v(-t)}<\frac{C_{1}}{v\left(\pi_{1}\right)-v(-m)}<1$

1) When $w(p) \in\left(0, \frac{C_{2}}{v\left(\pi_{2}\right)-v(-t)}\right)$, enterprises make production strategies according to the regulatory strategies of government departments, namely, $x>x^{*}, y=1$ and $x<x^{*}, y=0$. From above analysis we get government chose to regulate negatively, namely $x=0<x^{*}$, we have enterprises will choose traditional production. Therefore, $x=0, y=0$ is the evolutionary stable point.

2) When $w(p) \in\left(\frac{C_{2}}{v\left(\pi_{2}\right)-v(-t)}<\frac{C_{1}}{v\left(\pi_{1}\right)-v(-m)}\right)$, At this point, we can get from the above analysis,enterprises will choose low-carbon production strategy and government departments will choose negative regulation strategy. Therefore, $x=0$, $y=1$ is the evolutionary stable point.

3) When $w(p) \in\left(\frac{C_{1}}{v\left(\pi_{1}\right)-v(-m)}<1\right)$, enterprises choose low-carbon production, the strategy selection of government regulation depends on the production strategy of enterprise.namely $y>y^{*}, x=0$ and $y<y^{*}, x=1$. Due to $y=1>y^{*}$, we can get $x=0$, so government department chooses negative supervision. Therefore, $x=0, y=1$ is an evolutionary stable points.

To sum up,dynamic evolution strategy of enterprises and government in the above two situations are shown in Figure 1.

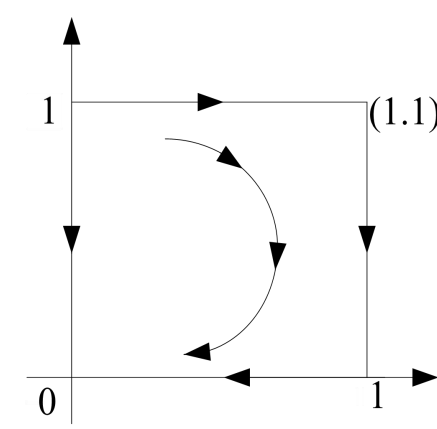

(a) $\quad w(p) \in\left(0, w_{1}(p)\right)$

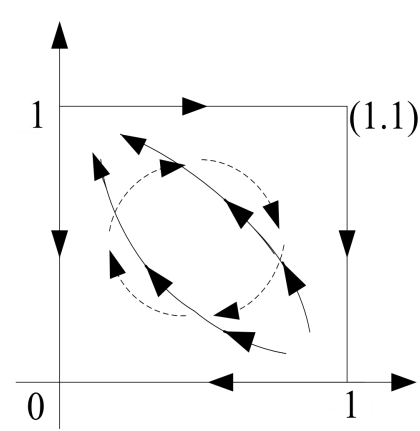

(b) $\quad w(p) \in\left[w_{1}(p), w_{2}(p)\right]$

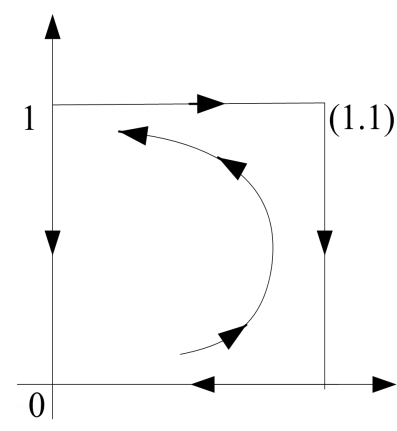

(c) $\quad w(p) \in[w 2(p), 1]$

FIG. 1 Dynamic diagram of the evolutionary game between government and enterprise

When the possibility of air quality deterioration is small, enterprises will choose traditional production methods, government will choose negative regulation. When the possibility of air quality deterioration is at a medium level, if the possibility of air 
quality deterioration is enough to promote the transformation of government departments to active regulation, but not enough to promote the transformation of enterprises to low-carbon production, the evolution strategy of government and enterprise will cycle indefinitely. When the probability of air quality deterioration reaches a level that prompts enterprises to transform to low-carbon production, but it is not enough to prompt government departments to change existing regulatory strategies, enterprises will choose low-carbon production,while government will choose negative regulation. When the probability of air quality deterioration is high, enterprises choose low-carbon production and the government chooses positive regulation in order to avoid huge losses, however, when the government realizes the transformation of enterprises to low-carbon production, they will change to negative regulation.

\subsection{Paradox analysis of carbon emission regulation incentive}

The government's punishment on enterprises traditional production and the government's loss of social benefits are described by graphic method. In Figure2, $\mathrm{X}$-axis represents the probability of traditional production by enterprises, $\mathrm{Y}$-axis represents the income from negative regulation by government departments.If the probability of enterprise traditional production is greater than $x_{0}$, the revenue of government departments is negative, so the government departments will choose positive supervision. Therefore, in order to ensure the maximization of income, the probability of enterprises' traditional production tends to $x_{0}$ rather than higher than $x_{0}$.

Government increase penalties on enterprises with traditional production in order to prompt enterprises to choose low-carbon production generally. The amount of fines will increase from $D$ to $D^{\prime}$, as shown in Figure 3, at this point, the enterprise's revenues will become negative under the same government regulatory probability, so the enterprise in order to obtain positive earnings must reduce the probability of traditional production. However, as the probability of traditional production decreases, government departments will gradually reduce the probability of active supervision, and enterprises will return to the probability level of original traditional production with the reduction of government supervision.

On the other hand, if we increase the punishment for negative supervision of government departments as shown in figure 2, The adds penalties make the loss of government departments for negative supervision from $t$ to $t^{\prime}$. This means that with the same degree of regulation, the returns to government departments become 
negative, so the government in order to keep its original income level will increase supervision strength, in turn, enterprise will decreases the probability of traditional production with the increase of intensity of government regulation.

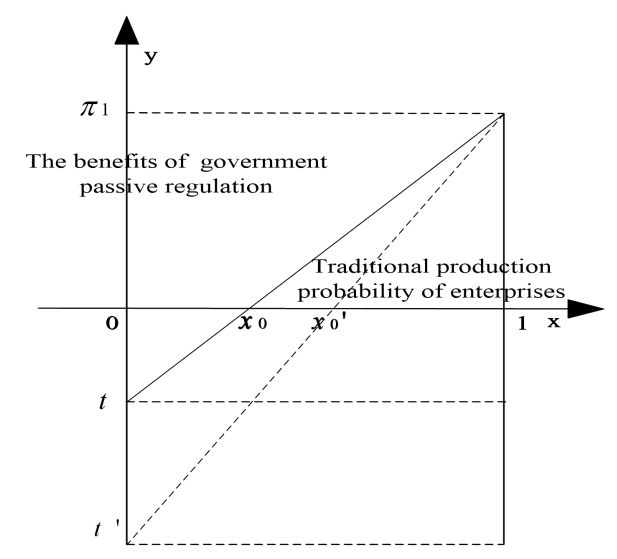

FIG.2 mixed strategies distribution of enterprise

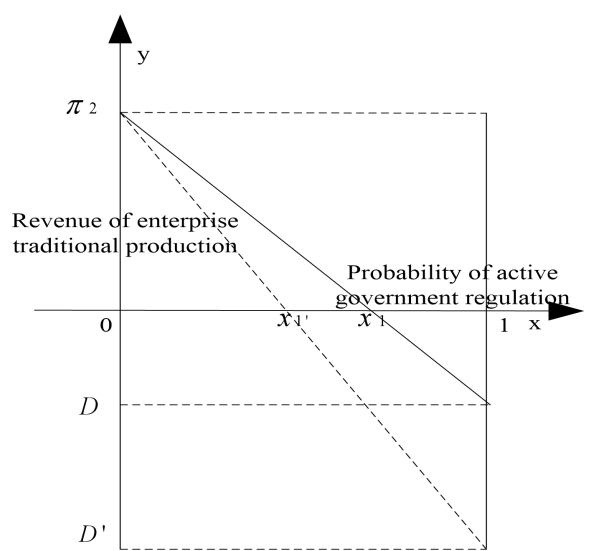

FIG.3 distribution of mixed strategies of government

Therefore, increasing the penalty on enterprises' traditional production mode can promote enterprises to transform to low-carbon production in the short term, but in the long run, it will only indirectly increase the probability of negative regulation by government departments, Eventually, the company reverts to its original mode of production.However, if increasing the punishment for ineffective supervision of government departments can make the government departments adopt active supervision strategies, this way will inhibit the possibility of enterprises adopting traditional production methods for a long time.

\section{Numerical simulation and result analysis}

\subsection{Carbon emission regulation case description}

Suppose that the government department chooses the positive supervision strategy with the income of $\pi_{1}=80$ and the supervision cost of input is $C_{1}=33$; the income of the enterprise chooses traditional production as $\pi_{2}=125$, the improvement cost of low-carbon production input as $C_{2}=60$; When the traditional production of the enterprise is found by the government regulatory department, the fine amount is $D=70$; When air quality deteriorates, the social benefit loss of government is $t=60$, the enterprise loss is $m=96$. All parameters are set in accordance with the basic assumptions and the reality of the model.According to equation (2):

The subjective perceived value of government regulators on gains and losses is : $v\left(\pi_{1}\right)=47.28, v(m)=73.42$; the Carbon emission enterprises' perception of the subjective value of gains and losses is as follows: $v\left(\pi_{2}\right)=70.03, v(t)=111.03$. 
Therefore, we can get the equilibrium point of air quality deterioration risk grade as : $w_{1}(p)=\frac{C_{1}}{v\left(\pi_{1}\right)+v(t)}=\frac{33}{47.28+73.42} \approx 0.27, \quad w_{2}(p)=\frac{C_{2}}{v\left(\pi_{2}\right)+v(m)}=\frac{60}{70.03-111.03} \approx 0.33$, from $w_{1}(p)<w_{2}(p)$, We have that this situation belongs to the second and hypothesis above. We assume that:

When $w(p) \in(0,0.27)$, it belongs to the situation of Low risk of air quality deterioration;

When $w(p) \in(0.27,0.33)$, it belongs to the situation of medium risk of air quality deterioration;

When $w(p) \in(0.33,1)$, it belongs to the situation of higher risk of air quality deterioration.

\subsection{Analysis of government and business evolutionary results}

Scenario1: Suppose the probability of air quality deterioration $p=0.1$, then $w(p)=0.19 \in(0,0.27)$ belongs to the game strategy under low risk of air quality deterioration. In this case, the replication dynamic equation of the government department and enterprise becomes:

$$
F(x)=x(1-x)(-22.49 y-10.51), \quad F(y)=y(1-y)(120.38 x-26.27) \text {. }
$$

Through MATLAB software simulation, we have the game strategy trend diagram of the government and the enterprise shown in FIG. 4:

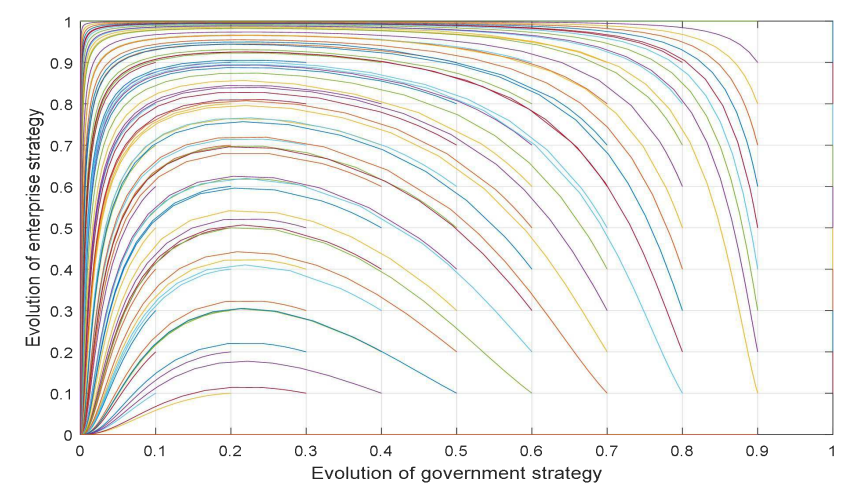

FIG. 4 evolution chart of government and enterprise strategies when air quality is good

We can be seen from figure 4 that $(0,0)$ is the evolutionary stable point,it indicate that when low risk of air quality deterioration, government evolve to negative regulation and enterprises evolve to traditional production.

Scenario 2: Suppose the probability of air quality deterioration $p=0.30$, then $w(p)=0.32 \in(0.27,0.33)$ belongs to the game strategy under medium risk of air quality deterioration. At this time, the replication dynamic equation of the government department and the enterprise becomes: 
$F(x)=x(1-x)(-35.09 y+2.09), \quad F(y)=y(1-y)(101.47 x-7.36)$.

Thus, we can get the game strategy trend diagram of government and enterprise shown in FIG.5:

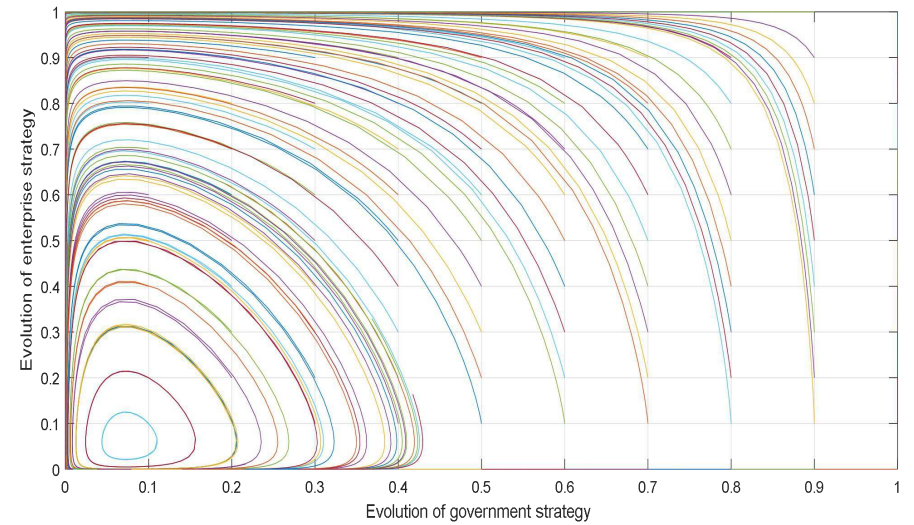

FIG. 5 Evolution chart of government and enterprise strategies when air quality is medium

We can get from figure 5 that the strategies of government and enterprise are determined to the strategies of the other side, their strategy has an infinite loop.

Scenario3: Suppose the probability of air quality deterioration $p=0.6$, then $w(p)=0.47 \in(0.33,1)$ belongs to the game under higher risk of air quality deterioration. The replication dynamic equation of government departments and enterprises becomes:

$$
F(x)=x(1-x)(-57.2 y+24.2), F(y)=y(1-y)(68.32 x+25.79)
$$

In this case, we have the evolution strategy trend between the government and enterprises, as shown in FIG. 6:

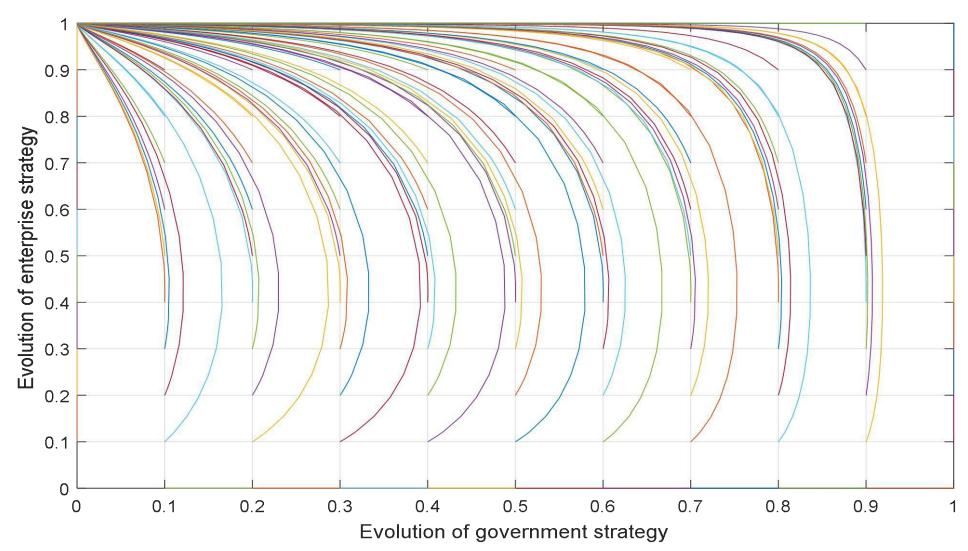

FIG. 6 Evolution chart of government and enterprise strategies when air quality is poor

From figure 6 ,we have that $(0,1)$ is the final evolutionary stable point. When faced the high risk of air quality deterioration, both government and enterprise will attach great importance to it. At this time,enterprises will choose low-carbon production strategy,the government will choose active regulation. However, when government realize the low-carbon production strategic of enterprises, they will turn 
to negative regulation.

\section{5 conclusion}

Aiming at the problem of carbon emission regulation, this paper considers the possibility of air quality deterioration caused by traditional production of enterprises, using prospect theory to correct the gains and losses of governments and enterprises, analyzes the strategic choice of governments and enterprises under different air quality deterioration probabilities.

Aiming at the problem of carbon emission regulation, this paper considers the possibility of air quality deterioration caused by traditional production of enterprises, using prospect theory to correct the gains and losses of governments and enterprises, analyzes the strategic choice of governments and enterprises under different air quality deterioration probabilities. The research results show that: 1) when the probability of deterioration in air quality is lower, the enterprises tend to choose the traditional of production, the government in the face of the enterprise's traditional production mode tend to be negative regulation. At this point, the government increases the amount of fines on traditional production, which can change the production behavior of enterprises in a short period, but in the long run it will make the government more passive regulation, and the enterprise back to the original production status. 2) When the probability of air quality deterioration is medium, the government and the enterprise will choose the opposite strategy, namely, the enterprise's production strategy change with the regulation strategy of the government department, when the enterprise's production strategy changes, the government's regulation strategy will also change. 3)When the probability of air quality deterioration is high, enterprises choose low-carbon production,government departments will choose active supervision at first.However, with the enterprise low carbon production behavior, the government gradually evolves from positive supervision to negative supervision.At this time, if the punishment for ineffective supervision of government is increased, the supervision of government departments will become more positive, which can effectively promote enterprises' low-carbon production behaviors in the long run. Thus, Strengthening the supervision, assessment, accountability, interview and other reward and punishment measures for government can effectively reduce the carbon emission behavior of enterprises. Therefore, the following suggestions are proposed:

1. Scientifically divide the financial rights of central and local governments in 
ecological governance. So that local governments have sufficient funds for ecological and environmental governance, no longer subject to the constraints of governance costs.

2. Establish a green GDP performance evaluation mechanism. The subjects, procedures and rewards and punishment measures should be clarified, government departments with good environmental governance performance will be rewarded and those with poor environmental governance performance will be punished.

3. Strengthen rewards and punishments for enterprises. Subsidies or preferential policies will be given to enterprises that actively engage in low-carbon production, penalties will be increased for those that violate the rules.

4.Timely disclosure of carbon emission information to the public. Make the masses establish low carbon environmental protection consciousness, at the same time encourage the public and industry association to participate in supervision, form external supervision mechanism.

Due to the government and enterprises may have different risk sensitivity coefficient and avoidance coefficient, this paper did not discuss in detail. Further analysis on the risk preference of government and enterprises, it is the work that needs to be carried out in the future research.

\section{Statement}

1."Funding : This study was funded by Shanghai philosophy and social Science planning project (grant number 2020BGL012 ).”

2."Conflict of Interest: The authors declare that they have no conflict of interest."

\section{References}

1. Banet,Catherine (2017) Effectiveness in Climate Regulation: Simultaneous Application of Carbon Tax and a Emissions Trading Scheme to the Offshore Petroleum Sector in Norway. Carbon \& Climate Law Review 11 (1) : 25-38.

2. Clarkson. P. M. and G,D.Richardson (2004) The Market Valuation of Environmental

Capital Expenditures by Pulp and Paper Companies. Accounting Review $79(2): 329-353$.

3. Du, S. F.,Wen,Z. T. and Malin Song (2016) Low-carbon production with low-carbon premium in cap-and-trade regulation. Journal of cleaner production 
134(15): 652-662.

4. Gu, B. J ,Zhang, X and LI,Y. L (2020) Perspective theory, psychological account and newsboy mediating effect. Journal of systems management 29 (3) : 549-560.

5. Gong, P. and Zhang, Y. Y (2020) Seller pricing Decision Model based on prospect theory. Journal of Systems Engineering 35(3): 340-353.

6. Jonathan Busch, Timothy J. Foxon, Peter G. Taylor (2018) Designing industrial strategy for a low carbon transformation. Environmental Innovation and Societal Transitions 29: 114-125.

7. Li, L. and Tao, F (2012) The Choice of Optimal Environmental Regulatory Intensity in China's Manufacturing Industry -From the Perspective of Green Total Factor Productivity. China's Industrial Economy 5: 70-82.

8. Li, P. P. and Sun, S. R (2018) Dynamic Pricing of Low Carbon Products and Carbon Emission Strategies Based on Government Support. Logistics Sci-Tech 41(8): 113-120.

9. Li,X. Y, LI, X. M (2017) Emotional Reference Points based Self-organizing Multi-agent Model of Route Choice . Journal of systems management 26(2): $259-267+276$.

10. Liu,W.B. and Han, T. K (2019) An Analysis of a Corruption incentive Paradox from the perspective of Game Theory. Management Research 1: 15-24.

11. Liu, J.G.and Wang, J. J (2018) Research on the supervision of hazardous chemicals in ports based on the level of safety risk. Systems Engineering Theory and Practice 38(5): 1141-1152.

12. N, J (2016) Analysis of Carbon Tax and Carbon Emission Trading Mechanism. Tax Research 4:46-50.

13. Porter, M. E. and C. V. D. Linde (1995) Toward a New Conception of the Environment Competitiveness Relationship. Journal of Economic Perspective 9(4): 97-118.

14. Qin, Y (2017) Designing of Low-Carbon Transformation Mechanism of Industry Based on Sector Interests Game. Ecological Economy 33(6): 64-68.

15. Zhang H (2014) Does environmental regulation improve carbon performance? Economic Management 12: 166-175.

16. Sinn,Hans-Werner (2008) Public policies against global warming: a supply side approach.International Tax and Public Finance 15(1): 360-394. 
17. Tang, S.Z.,Xiao, T. J.and Liu, Y.Z (2020) Supply chain pricing and carbon emission reduction decisions considering social responsibility. China management science 28(4): 99-108.

18. Tang, S.Z.,Xiao, T. J.and Liu, Y.Z (2020) Supply chain pricing and carbon emission reduction decisions considering social responsibility. China management science 28(4): 99-108.

19. Wang, T. T, Wang, D. P (2020) Dynamic coordination strategy of supply chain cooperation on emission reduction and low carbon publicity under government subsidies. Operations research and management 29(8): 52-61.

20. Wang, Z.Y. and Yang, X.L (2020) An evolutionary game analysis of panic buying events considering the perceived value of the public. China management science 28 (3): 71-79.

21. Zhao, L. J., LI, X. and Zhu, D. L (2005) On Environmental Law Enforcement in China from the Perspective of Incentive Paradox. Soft Science 4: 52-55.

22. Zhao, Z.B. and Man, Q.P (2015) Evolutionary Game Analysis of risk management behavior of Major Infrastructure Projects based on prospect Theory. Journal of Systems Management 27(1): 109-117. 